\title{
C. difficile is overdiagnosed in adults and a commensal in infants
}

\author{
Pamela Ferretti ${ }^{1}$, Jakob Wirbel ${ }^{1, \mathrm{x}}$, Oleksandr M Maistrenko ${ }^{1,2, \mathrm{x}}$, Thea Van Rossum ${ }^{1, \mathrm{x}}$, Renato Alves ${ }^{1}$, \\ Anthony Fullam ${ }^{1}$, Wasiu Akanni ${ }^{1}$, Christian Schudoma ${ }^{1}$, Anna Schwarz ${ }^{1}$, Roman Thielemann ${ }^{1}$, Leonie \\ Thomas $^{1}$, Michael Kuhn ${ }^{1}$, Georg Zeller ${ }^{1}$, Thomas SB Schmidt ${ }^{1}$ and Peer Bork ${ }^{1,3,4,5}$
}

Affiliations:

1 European Molecular Biology Laboratory, Structural and Computational Biology Unit, 69117 Heidelberg, Germany

2 Present address: Royal Netherlands Institute for Sea Research (NIOZ), Department of Marine Microbiology \& Biogeochemistry, 1797 SZ, 't Horntje (Texel), Netherlands

3 Max Delbrück Centre for Molecular Medicine, Berlin, Germany

4 Yonsei Frontier Lab (YFL), Yonsei University, Seoul 03722, South Korea

5 Department of Bioinformatics, Biocenter, University of Würzburg, Würzburg, Germany

$x$ These authors contributed equally to this work.

*Correspondence: sebastian.schmidt@embl.de and Bork@embl.de

\section{Abstract}

Clostridioides difficile is an urgent threat in hospital-acquired infections world-wide, yet the microbial composition associated with $C$. difficile, in particular in $C$. difficile infection (CDI) cases, remains poorly characterised. Here, we analysed 534 metagenomes from 10 publicly available CDI study populations. While we detected C. difficile in only $30 \%$ of CDI samples, multiple other toxigenic species capable of inducing CDI-like symptomatology were prevalent, raising concerns about CDI overdiagnosis. We further tracked C. difficile in 42,814 metagenomic samples from 253 public studies. We found that $C$. difficile prevalence, abundance and association with other bacterial species is age-dependent. In healthy adults, $C$. difficile is a rare taxon associated with an overall species richness reduction, while in healthy infants $C$. difficile is a common member of the gut microbiome and its presence is associated with a significant increase in species richness. More specifically, we identified a group of species co-occurring with $C$. difficile exclusively in healthy infants, enriched in obligate anaerobes and in species typically found in the gut microbiome of healthy adults. Overall, gut microbiome composition in presence of $C$. difficile in healthy infants is associated with multiple 
parameters linked to a healthy gut microbiome maturation towards an adult-like state. Our results suggest that $C$. difficile is a commensal in infants, and that its asymptomatic carriage is dependent on the surrounding microbial context.

Keywords: Clostridioides difficile, metagenomics, CDI, antibiotic-associated diarrhea, infant microbiome, overdiagnosis

\section{Introduction}

Clostridioides difficile (previously Clostridium or Peptoclostridium difficile) was first isolated in 1935 from the stools of healthy infants ${ }^{1}$, but later identified as a causative agent of pseudomembranous colitis ${ }^{2}$, a severe condition with potentially life-threatening outcomes. While not all $C$. difficile strains are toxigenic, $C$. difficile toxin $A(\operatorname{Tcd} A)$ and toxin $B(T c d B)$ can damage the host by increasing intestinal permeability and promoting intense inflammation ${ }^{3}$, a condition referred to as $C$. difficile infection (CDI). $C D I$ is typically characterised by diarrhea and/or pseudomembranous colitis and often unresponsive to repeated antibiotic treatments. CDI has an increasing clinical relevance (at an estimated annual economic burden of US\$6.3 billion in the US alone ${ }^{4}$ ) and is considered one of the most urgent threats in hospital-acquired infections ${ }^{5}$. C. difficile has primarily been studied as a pathogen, with a strong focus on CDI aetiology ${ }^{6}$, mechanisms of $C$. difficile toxicity ${ }^{3}$ or efficacious therapies ${ }^{7}$. However, an increasing number of other enteropathogens has been linked to antibiotics-associated diarrhea (AAD) with similar, and sometimes indistinguishable, symptomatology to $\mathrm{CDI}^{8-10}$, making the correct diagnosis, estimation and management of CDI particularly challenging.

Far less is known about asymptomatic $C$. difficile carriage among the healthy population, as sentinel studies are often limited in sample size and geography ${ }^{11,12}$. Gut microbial composition associated with $C$. difficile presence in healthy subjects, in particular in infants where $C$. difficile is highly prevalent ${ }^{13}$, remains poorly understood.

Here we leverage public shotgun metagenomic data to characterise the gut microbial signature associated with CDI in adult and elderly patients and to quantify other AAD-associated species with similar signatures. We then contextualise our analysis by tracing $C$. difficile in a broad multihabitat collection of 42,814 metagenomes. We investigate $C$. difficile prevalence, relative abundance and its associated microbial community structure and composition in the human gut over lifetime, with particular focus on healthy infants, where several lines of evidence point to $C$. difficile being a commensal and a hallmark species of healthy infant gut microbiome maturation. 


\section{Results}

\section{C. difficile is detectable in only $30 \%$ of patients with a CDI diagnosis}

We first conducted a metagenomic meta-analysis of the gut microbiome associated with CDI, analysing 534 fecal samples across 10 geographically diverse study populations ${ }^{14-22}$, including patients diagnosed with CDI, diseased subjects without CDI (D-Ctr, see Methods), and healthy subjects (H-Ctr) (Figure 1A and Supplementary Table 1).

Using mOTUs-based species-level taxonomic profiling ${ }^{23}$, C. difficile was detectable in just $30 \%$ of $\mathrm{CDI}$ samples at established metagenomic detection limits ${ }^{23}$ (Figure 1A), with considerable variance across study populations (9.2\%-92.3\%), and with much lower prevalence among diseased $(2.6 \%)$ and healthy $(1.1 \%)$ controls (Supplementary Figure 1A). C. difficile toxin genes $t c d A$ and $t c d B$ were detectable in about half of $C$. difficile-positive CDI metagenomes, but not in any $C$. difficile-negative CDI samples nor controls, indicating a lower sensitivity of toxinbased detection (Figure 1A, see Methods). We confirmed that marker gene-based $C$. difficile detection was not limited by sequencing depth (linear mixed effect model $p=0.35$, ANOVA, adjusted $p=0.54 ; R^{2}=0.01$, and that detection was not skewed towards the theoretical metagenomic detection limit of $10^{-5}$ relative abundance ${ }^{24}$. Neither species richness (Figure 1B) nor composition (Supplementary Figure 2) differed between C. difficile-positive and -negative CDI samples, suggesting that $C$. difficile presence was not associated with characteristic community level shifts in these generally disbalanced microbiomes.

\section{The microbiome of diagnosed CDI patients is characterised by an enrichment of enteropathogens beyond $C$. difficile}

Several other species were enriched in prevalence or abundance in CDI patients relative to controls (Figure 1C, Supplementary Figure 3). Among these, we identified several opportunistic enteropathogens known to induce antibiotic-associated diarrhea (AAD), resulting in a CDI-like diarrheal symptomatology ${ }^{9,10,25-29}$, such as Klebsiella oxytoca, Citrobacter amalonaticus, Clostridium innocuum, Clostridium perfringens, Staphylococcus aureus, Enterococcus faecalis, Enterobacter cloacae, and Pseudomonas aeruginosa. In particular, C. innocuum was highly prevalent (63.2\%) in CDI samples, and significantly enriched relative to both healthy and diseased controls (Figure 1C, Wilcoxon test $p=8.2 \times 10^{-16}$ and $p=4.3 \times 10^{-3}$, respectively). $94 \%$ of all CDI samples contained at least one of these other AAD species ( $C$. difficile excluded) with variable prevalence across study populations (Fisher's test, $\mathrm{p}=2.2 \times 10^{-16}$, 
OR=29.62; Supplementary Figure 1A and 1B). Presence and potential toxigenicity of several enteropathogens was further confirmed by the detection of characteristic toxin genes (see Methods) that were likewise enriched in CDI samples (Figure 1D). In particular, enteropathogenic E. coli and S. flexneri were, either alone or in combination with other species, the most common species with toxin genes. Overall, CDI samples, independently of $C$. difficile presence, were significantly enriched $\left(p=2.22 \times 10^{-16}\right)$ in species carrying toxin genes in terms of cumulative relative abundance, indicating that CDI symptomatology could be entirely or partially driven by other enteropathogens than $C$. difficile.

To integrate these univariate associations of individual species into characteristic and predictive multi-species signatures of CDI, we next trained a series of LASSO-regularised logistic regression models in a leave-one-study-out validation approach (see Methods). As expected, $C$. difficile was the most predictive species for CDI samples, although the enrichment of other putative AAD enteropathogens (such as $S$. aureus or $C$. perfringens) was also highly characteristic, in particular in CDI samples where C. difficile was not detectable (Figure 2A). Moreover, several oral species (such as Veillonella parvula, Veillonella atypica or Rothia dentocariosa) were enriched in $\mathrm{CDI}$ gut metagenomes, implying increased oral-intestinal microbial transmission ${ }^{30}$ among these patients. Enrichment of common probiotics (Lactobacillus casei, L. plantarum or L. fermentum) in CDI is likely due to the widespread use of probiotic therapy among these patients ${ }^{31,32}$ or to the increased antibiotic resistance of these species ${ }^{33}$.

Using these models, CDI samples were distinguishable from diseased and healthy controls with high accuracy, at an area under the receiver operating characteristics curve (AUROC) of 0.78, ranging between 0.56 and 0.98 depending on the study population (Figure 2B), in line with a previous $16 \mathrm{~S}$-based investigation ${ }^{34}$. Model performance moderately improved when considering only healthy controls (overall AUROC $=0.81$ ) (Figure 2B). To assess the importance of $C$. difficile to predicting CDI state, we next re-trained models under explicit exclusion of $C$. difficile. Interestingly, this did not lead to a noticeable reduction in accuracy (Figure 2B), indicating that the presence or enrichment of $C$. difficile was indeed not an essential defining feature of CDI samples. Rather, $C$. difficile appeared to be just one of several AAD species characterising the CDI-associated microbiome in varying constellations, forming a loose clique instead of fixed cooccurring species groups. 


\section{Extensive asymptomatic carriage of $C$. difficile across age and geography}

Given that $C$. difficile appears to have a much lower prevalence in CDI patients than anticipated, we next studied its prevalence in an extended collection of 253 publicly available studies, for a total of 42,814 shotgun metagenomic samples (Supplementary Table 2), including healthy and diseased subjects of all ages, with samples from multiple body sites, hosts, environments and geographic locations (Figure 3A and 3B). Results from this point onwards refer to this extended collection of samples.

C. difficile was detected almost exclusively in human and animal feces $(5.9 \%$ and $1.7 \%$ prevalence, respectively) with sporadic detection in hospital surfaces $(0.19 \%)$, the respiratory tract of diseased patients $(0.37 \%)$ and freshwater $(0.88 \%)$ (Figure $3 \mathrm{C}$ and Supplementary Table 3). C. difficile was also detectable in the colon, ileum and cecum lumen, and biopsies from the colon and rectal mucosa of healthy subjects. We again confirmed in this larger dataset that there is no relationship between $C$. difficile detection and sequencing depth (linear mixed effect model $p=0.36$; logistic regression, ANOVA adjusted $p=0.44$, Supplementary Figure 4A). Nevertheless, we adjusted all subsequent analyses for total sequencing depth.

In healthy human populations, both $C$. difficile prevalence and relative abundance in fecal samples varied considerably across age ranges. Infants aged one year or younger showed by far the highest $C$. difficile carriage, up to $76.5 \%$ prevalence at the age of 8 to 10 months (Figure 4A), at an average relative abundance of $1.1 \%$ (and up to $50 \%$ in a two-weeks-old healthy preterm infant). C. difficile prevalence remained elevated (44.5\%) between 1 and 4 years of age, in contrast to previous reports ${ }^{35,36}$. Among older individuals, $C$. difficile was increasingly rare, found in less than $1.06 \%$ of the healthy population $(7.6 \%$ in adolescents, $0.92 \%$ in adults) (Figure 4A and Supplementary Table 3 ) at average abundances of $0.2 \%$, suggesting a considerably lower asymptomatic carriage among adults than previously estimated (up to $15 \%{ }^{37}$ ). C. difficile carriage varied across geography (Supplementary Figure 4B) and was lower in non-westernised populations, at $4.76 \%$ and $0.35 \%$ in infants and adults, respectively.

\section{C. difficile is enriched upon antibiotic treatment}

Diseased or antibiotics-treated subjects exhibited consistently increased $C$. difficile carriage rates across all age groups (Figure 4A). Prevalence was highest among infants taking antibiotics $\left(81.1 \%\right.$, relative abundance $\left.2.22^{-2}\right)$ and infants suffering from cystic fibrosis $(78.7 \%$, 
$4 \times 10^{-3}$ ) or neonatal sepsis/necrotizing enterocolitis (55.3\%, 3.2 $\times 10^{-2}$; Supplementary Figure 5). An elevated $C$. difficile asymptomatic carriage rate in cystic fibrosis patients is well documented $^{38}$ and may be the result of long-term antibiotics usage and frequent exposure to the hospital environment ${ }^{39}$. Among adolescents, adults and elderly, $C$. difficile prevalence was highest in subjects taking antibiotics (on average $43.6 \%$ across age groups, relative abundance $\left.4.4^{-3}\right)$, followed by patients suffering from CDI (30\%, see above) or undefined diarrhea (25.9\%). Inflammatory bowel diseases, liver diseases, diabetes, and various forms of cancer were likewise associated with an increased $C$. difficile carriage relative to the healthy background population (Supplementary Figure 5).

\section{C. difficile is a frequent commensal in the healthy infant gut}

C. difficile was among the most prevalent species in infants during the first year of life. Carriage was increased in infants born via C-section (30.16\%; logistic regression, adjusted ANOVA $\mathrm{p}=2.1 \times 10^{-5}, \log \mathrm{OR}=3.1 \times 10^{-5}$ ) compared to age- and health status-matched vaginally born infants (24.94\%) (Figure 4B and Supplementary Figure 6). Likewise, prevalence was moderately increased among pre-term (36.17\%; $p=3.3 \times 10^{-4}$, logOR $=2.5 \times 10^{-5}$ ) compared to full-term healthy infants (25.62\%) (Figure 4C and Supplementary Figure 6). Exclusively formula-fed infants were more frequently colonised by $C$. difficile $\left(43.18 \% ; p=4.8 \times 10^{-9}\right.$, logOR $\left.=5 \times 10^{-5}\right)$, compared to partially or exclusively breastfed infants during the first 6 months of life $(14.23 \%$ and $8.6 \%$ respectively) (Figure 4D and Supplementary Figure 6), in line with previous reports ${ }^{40,41}$.

C. difficile was not maternally acquired during birth: across 820 mother-infant pairs included in the dataset, we found no single case of vertical transmission of $C$. difficile from any of the maternal body sites investigated (gut, vagina and oral cavity). Rather, $C$. difficile was likely sourced from the environment during early life, in line with previous observations on Clostridia ${ }^{42}$. Leveraging longitudinal data, we observed that the first appearance of $C$. difficile was not arbitrarily distributed over the first year of life, but instead concentrated in two defined time windows, between the $2^{\text {nd }}-4^{\text {th }}$ and $8^{\text {th }}-10^{\text {th }}$ months of age (Supplementary Figure 7 ). The first interval coincided with the increased strain influx from environmental sources to the infant gut reported in a previous study ${ }^{43}$, probably due to the start of mouthing. The second interval, dominated by samples from the UK, overlaps with the paid maternity leave length in that country $^{44}$, suggesting that it could be due to the start of day care. C-section and premature birth were associated with increased rate of $C$. difficile appearance, compared to vaginally born and 
full-term infants (Supplementary Figure 7). Together these results suggest that $C$. difficile is acquired from the environment, and that increased exposure to novel environmental sources increases the chances of $C$. difficile acquisition. In addition, during the first year of life, the gut microbiome of healthy infants colonised with $C$. difficile was significantly more similar, in terms of composition, to that of their mothers (Supplementary Figure 8).

These vast differences in $C$. difficile carriage across age did not translate into differential toxin burdens. $C$. difficile toxin genes were detectable in metagenomes of healthy $C$. difficile-positive infants $(22.6 \%)$ and adults $(26.8 \%)$ at similar rates (Supplementary Figure 9 ).

\section{C. difficile occurs in distinct biotic and physiological contexts in infants and adults}

The $C$. difficile-associated microbiome varied considerably across age ranges. $C$. difficile carriage was associated with a significantly decreased overall species richness in subjects above 1 year of age, independently of the health status (Figure 4E). However, in healthy infants we observed the opposite trend: species richness was higher in subjects carrying $C$. difficile (Figure 4E and Supplementary Figure 10A), irrespective of delivery mode, gestational age or geography (Supplementary Figure 10B and 10C). Species richness was also elevated in $C$. difficile-positive diseased infants, albeit to a lesser degree. In line with these trends, $C$. difficile carriage was associated with higher community evenness (lower dominance of individual species) in infants, but a more uneven community structure in adults (Supplementary Figure 10D).

To further characterise how the biotic context of $C$. difficile in the gut changes over lifetime, we studied its co-occurrence with other intestinal species, stratified by age group and health status. We broadly distinguished two groups of species based on their co-occurrence relationships with C. difficile (Figure 5), independently of the presence of its toxin genes. The first set of species ("Group 1") consistently co-occurred with C. difficile across all age groups. Part of this group are Clostridium paraputrificum and Clostridium neonatale (Supplementary Figure 11A), in line with previous reports ${ }^{45}$, an association that was conserved also in animal hosts (Supplementary Figure 11B). Group 1 was enriched in facultative anaerobic or aerobic species and comprised several known AAD enteropathogens, such as $K$. oxytoca, $C$. perfringens, $C$. amalonaticus or $C$. innocuum. A second set of species ("Group 2") co-occurred with $C$. difficile exclusively in healthy infants, but showed strong avoidance patterns in later life stages and, to a lesser extent, 
in diseased infants. Group 2 was characterised by obligate anaerobic species typical of the healthy adult gut microbiome and included several common butyrate producers (such as Roseburia sp., Faecalibacterium prausnitzii or Anaerostipes hadrus) (Figure $\mathbf{5}$ and Supplementary Figure 12).

To investigate whether healthy infants were associated with specific $C$. difficile strains, we performed an analysis of metagenomic single nucleotide variants (SNVs). Despite the distinct biotic context associated with $C$. difficile presence in healthy infants at the species level, $C$. difficile strain populations found in this group did not cluster separately from those identified in other age groups and health status, with consistent patterns of toxin-gene carriage (Supplementary Figure 13). Therefore, the general asymptomatic nature of $C$. difficile carriage in healthy infants is likely determined by its surrounding microbial community and host age, rather than specific strain groups.

\section{Discussion}

C. difficile has been predominantly studied in the context of its pathogenicity, but our analysis suggests that considering $C$. difficile exclusively as a pathogen is falling short. $C$. difficile's role in causing antibiotics-associated diarrhea (AAD) or pseudomembranous colitis (PMC) may have been overestimated as our results strongly suggest that symptoms can also be explained by other AAD or PMC associated species, which would indicate CDI over-diagnosis in clinical practice. We detected fecal $C$. difficile in just $30 \%$ of cases diagnosed with CDI. This is consistent with the absence of $C$. difficile among $\mathrm{CDI}$ samples reported in previous $16 \mathrm{~S}$ rRNA $^{45,46}$ and WGS metagenomic studies ${ }^{14,47}$, even when combined with laboratory-based tests $^{46}$. Although the sensitivity of our analysis is dependent on metagenomic detection limits in principle, we found that variance in sequencing depth did not explain the observed variability in C. difficile detection. While it is possible that $C$. difficile might have been successfully eradicated by the antibiotic treatments following the CDI diagnosis but anteceding the sampling, the CDI samples included in this analysis were labelled as CDI in the original studies. In this context, we hypothesise that the under-detection of $C$. difficile among CDI patients is likely due to a lack of diagnostic specificity. Proper CDI diagnosis is surprisingly challenging in current health systems, with only marginal consensus on the use of the vast array of protocols and diagnostic algorithms available in the clinical practice. Diarrheal symptoms, often the initial diagnostic prompt, are not 
specific to $\mathrm{CDI}^{48-51}$ and pseudomembranous colitis, long thought to be characteristic (and often eponymously referred to as " $C$. difficile colitis"), may indeed be caused by other enteropathogens, such as $C$. innocuum or $E$. coli ${ }^{8,9,52}$. Additional challenges include the large variability in sensitivity and specificity in diagnostic laboratory tests ${ }^{40,53-62}$, contradicting results between cultivation and toxin detection assays ${ }^{63}$ and an excessive reliance on single molecular tests $^{64}$. Recommended guidelines discourage stand-alone tests for CDI diagnosis and recommend a two-step procedure ${ }^{53,57}$, but due to costs, test availability, capacity and turnaround times, diagnostic algorithms vary between hospitals and often deviate from recommended procedures $^{58}$. In our CDI meta-analysis, almost two thirds $(62.5 \%)$ of the studies with published CDI diagnostic protocols did deviate from recommended best practices. The use of multiple alternative protocols within studies, combined with the lack of published per-sample diagnostic information, prevented further investigation of variability in $C$. difficile detection rates between different diagnostic protocols.

Simultaneous colonisation of multiple enteropathogens is very common among CDI patients ${ }^{65,66}$. We found several species known to induce AAD or PMC enriched in CDI samples, along with their characteristic toxin genes, independently of $C$. difficile presence. In particular, at least one of these species is present in $90 \%$ of $C$. difficile negative CDI samples (toxins were detected in $43 \%$ of samples). While $C$. difficile was the most individually characteristic species in CDI, it was not an essential input to microbiome-based classification models accurately predicting the disease. Enteropathogens like C. perfringens, C. innocuum, S. aureus, E. coli or S. flexneri, among others, may contribute to or indeed drive clinical manifestations of $\mathrm{CDI}^{9,10,25-27,29}$. Our data strongly suggests that differential diagnosis against multiple enteropathogens may stratify patients with CDI-like symptoms, towards adapted therapeutic interventions. Disentangling the real burden of $C$. difficile from other AAD species could also aid its classification, which remains inconsistent, with most studies classifying $C$. difficile as a pathogen, some as an opportunistic pathogen, and few others as a pathobiont. This is also attributed to the limited study of $C$. difficile in healthy individuals and hence potential commensal features. To address this, we tracked $C$. difficile across an extensive set of 42,814 metagenomes sampled from multiple habitats and hosts. Samples from the human gut $(n=28,347,66,2 \%)$ covered a wide range of subject ages, disease states and geographic origins. C. difficile was very common among healthy and diseased infants, peaking at $>75 \%$ prevalence at 8-10 months of age. On average C. difficile was present in $25 \%$ of healthy infants aged 1 year or younger, in line with previous studies $^{35,36}$. 
In healthy infants, we could associate the occurrence of $C$. difficile with an increased resemblance to the maternal gut microbial community, general increase in microbiome richness and with several obligate anaerobic commensals, buyrate producers and a generally more adult-like microbiota, indicating that $C$. difficile may be a transient hallmark of healthy gut microbiome maturation. Indeed, an increase in species richness and number of obligate anaerobes are among the community-wide changes that the infant gut microbiome undergoes during its healthy development, as shown in several previous studies ${ }^{43,67-70}$. In contrast, $C$. difficile presence in adulthood was associated with decreased microbiome richness and an enrichment in facultative and obligate aerobes. Increased oxygen tolerance in the adult gut has been associated with dysbiotic states found in IBD patients and cases of Salmonella proliferation ${ }^{71,72}$. We found multiple lines of evidence suggesting that the transition between these apparent dichotomic states (commensal in infancy, pathobiont in adulthood), begins at around $10( \pm 2)$ months of age. We hypothesise that $C$. difficile could be an indicator species of a larger-scale and fundamental change taking place in the microbial ecology of the gut.

C. difficile toxin genes were likewise prevalent among infants, in line with previous reports ${ }^{13,73}$, indicating asymptomatic carriage of toxigenic strains. Indeed, $C$. difficile testing is generally discouraged by pediatric guidelines even in presence of diarrheal symptoms ${ }^{40,74}$ and toxin concentration may not be a reliable proxy for inferring disease and its severity ${ }^{73,75}$. The apparent protection from CDI symptoms has been ascribed to a lack of toxin receptors in the immature infant gut, although this early hypothesis ${ }^{76}$ has since been called into question ${ }^{77,78}$. The biotic context of $C$. difficile suggests additional or alternative explanations. We observed a significant enrichment in butyrate producers co-occurring with $C$. difficile in healthy infants, but not later in life nor in diseased infants. High levels of butyrate have been linked to inflammation inhibition, regulation of cell-to-cell tight junctions and increased mucin production (and therefore mucosal layer thickness and integrity ${ }^{79,80}$, all considered protective against $C$. difficile toxins ${ }^{81}$.

Our analyses are descriptive and arguably inherently limited by the underlying metagenomic data, such as DNA extraction bias against endospores ${ }^{82}$, incomplete correlation of gene dosage and activity $^{64}$ or general detection limits due to finite sequencing depth. Moreover, although some longitudinal data was available, time series were too sparse to infer causal relationships, such as e.g. the possible role of $C$. difficile in a partially deterministic community succession in the infant gut. To our knowledge, ours is the largest single-species metagenomic survey to date, 
demonstrating the utility of metagenomic meta-studies as a nuanced approach to gut microbial ecology, beyond $C$. difficile. Overall, our results suggest that the ability of $C$. difficile to induce disease may be context-dependent and multifactorial (i.e influenced by the rest of the microbiome composition, toxin presence and host age). Further study of $C$. difficile's association with health outcomes should therefore adopt a more holistic view of the ambivalent role of this species over the human lifespan.

\section{Online Methods}

\section{Data overview}

\section{CDI meta-analysis}

In the CDI meta-analysis, out of 534 samples in total (from as many subjects, with an average age of $60 \pm 19.88$ years), 234 were identified as CDI, 114 as diseased controls and 186 as healthy controls. Samples from subjects diagnosed with CDI in the original study population were classified as CDI in our meta-analysis. Samples were identified as controls in any of the following cases: (i) H-Ctr: healthy subject, reported as "control" in the metadata of the original study population; or (ii) D-ctr: diseased subject, that either had diarrheal symptoms but negative CDI diagnostic outcome, or was diarrhea-free but diagnosed for a disease other than CDI. The majority of the samples in the latter group (D-Ctr) belong to hospitalised subjects.

\section{Global meta-analysis}

We analysed a total of 42,814 publicly available metagenomic samples (collectively $>220 \mathrm{Tbp}$ ) from 253 different studies (see Supplementary Table 2). The collection includes samples from 84 countries, 35 animal species and 6 different human body sites: gastrointestinal tract (stools, rectal swabs and biopsies), vagina, skin, oral cavity, respiratory tract and human milk. The selected environmental samples come from potentially faecally contaminated habitats, such as wastewater, freshwater, indoor surfaces, soil and polluted harbor marine waters. 


\section{Data download}

Metagenomes publicly available on the $22^{\text {nd }}$ of April 2021 were downloaded using fetch-data ${ }^{83}$. Only shotgun metagenomic samples sequenced using Illumina platforms have been included. No minimum number of samples per study or minimum sequencing depth threshold was applied during data download.

\section{Metadata curation}

Manually curated metadata for each dataset include: health status, age, geography (country and continent name), westernised lifestyle or not, diagnosis for $C$. difficile infection (CDI), use of antibiotics, delivery mode, premature birth (pre-term or full-term), and sex. Subjects were categorised by age group, defined as follows: $0 \leq$ infant $\leq 12$ months, 1 year $<$ child $\leq 10$ years, 10 years < adolescent $\leq 18$ years, 18 years < adult $\leq 65$ years, and elderly > 65 years of age. If a specific age was not available, a range of age was provided in alternative. Given the broad range of perturbations in the gut microbiome associated with antibiotics intake, we ad-hoc defined "diseased" samples as any sample taken from a subject with any medically diagnosed disease or syndrome and/or intake of one or more antibiotics at the time of the sampling.

\section{Filtering on read count}

Two consecutive read count filtering steps were performed on all samples: (i) samples with zero reads were discarded $(n=1,091)$ and (ii) samples with less than 59 reads, corresponding to $95 \%$ ile calculated on the remaining samples, were discarded $(n=2,083)$. An additional third read count filtering was performed only on human gut stool samples, corresponding to $99 \%$ ile, removing samples with less than 100 reads ( $n=138$ samples). The resulting filtered dataset included 39,502 samples, of which 26,784 were human fecal metagenomes.

\section{Identification of timeseries-representative samples}

Out of 26,784 samples, 24,864 had subject-level metadata and were associated with 12,402 unique subjects. For 3,576 subjects, multiple timepoints were available. The mean number of timepoints per subject was 2 (3.18 for infants, 1.68 for children, 3.56 for adolescents, 1.68 for adults and 1.34 for elderly), with a maximum of 205 time points per subject.

In order to avoid under- or over-estimating $C$. difficile prevalence, only one sample per time series was used in cross-sectional analyses. We distinguished three cases for each time series: 
(i) C. difficile observed in all samples. In this case, the sample with the highest $C$. difficile read count is selected as representative and the corresponding subject was considered $C$. difficile positive.

(ii) C. difficile observed in none of the samples. In this case, the sample of the first time point was selected as representative and the corresponding subject was considered $C$. difficile negative.

(iii) C. difficile observed in only some samples. In this case, the sample with the highest $C$. difficile read count was selected as representative and the corresponding subject was considered $C$. difficile positive.

No subject metadata were available for 1,920 samples. In this case, we assumed one sample per subject. One representative sample for each time series was used for all downstream analyses, if not specified otherwise. The timeseries dereplication procedure described above has also been applied to the CDI study populations before downstream analysis.

\section{Metagenomic data processing}

\section{Taxonomic profiling}

Metagenomes were taxonomically profiled at the species level with mOTUs $v 2.0^{23}$, requiring the confident detection of at least two taxonomic marker genes. All data analyses were conducted in the R Statistical Computing framework v3.5 or higher. Only $C$. difficile positive samples were considered for $C$. difficile relative abundance estimation.

\section{Microbiome diversity}

Local community diversities ('alpha' diversities) of human gut samples were estimated by iteratively rarefying taxonomic count tables to 100 marker gene-mapping reads and computing average Hill diversities at $\mathrm{q}=0$ (species richness), $\mathrm{q}=1$ (exponential Shannon entropy) and $\mathrm{q}=2$ inverse Simpson index), as well as evenness measures as ratios thereof. Unless otherwise stated, results in the main text refer to taxa richness. Differences in alpha diversity were tested using ANOVA followed by post hoc tests and Benjamini-Hochberg correction, as specified in the main text.

\section{Prevalence and abundance estimations of $C$. difficile}

Prevalence estimations of $C$. difficile over life time were based on human gut samples (stools, rectal swabs and biopsies) where the precise subject age in months was available (samples 
with missing or too broad or age ranges were discarded). Both $C$. difficile mOTUs2.0 ("ref_mOTU_0051" and "ref_mOTU_0052") were considered in downstream analysis. Abundance estimations included only $C$. difficile human stool samples with precise age metadata.

\section{Species co-occurrence analysis}

Human gut stool samples, with at least 100 reads per sample and with known age group and health status were considered for this analysis. One representative sample per time series was considered. Fisher's exact test, followed by Benjamini-Hochberg correction, were applied to identify co-occurring species. Meaningful positive association was identified for species with adjusted p-value $<0.05$ and logarithm of the odds ratio $>1$, while meaningful negative association was identified for species with adjusted p-value $<0.05$ and logarithm of the odds ratio $<-1$.

\section{Machine learning modelling}

L1-regularised LASSO logistic regression models to predict CDI status were built using the SIAMCAT R package ${ }^{84}$ with 10-fold cross-validation. For this analysis, we focused on the subset of $10 \mathrm{CDI}$ or diarrhea-associated datasets (Supplementary Table 1) and then trained two different sets of models: one set of models to distinguish CDI samples and samples from healthy controls (excluding controls from diseased subjects) and another set of models to distinguish CDI samples and any type of control samples. In order to minimise overfitting and to counter batch effects ${ }^{84}$, we pooled datasets across studies in a leave-one-study-out approach. In short, all except one study were jointly processed to train a LASSO model that was then used to predict the left-out study. Additionally, to check if the microbial signature for CDI was independent of $C$. difficile, we trained another set of models with the same cross-validation splits but excluded $C$. difficile from the feature table. Feature weights were extracted from the models, normalised by the absolute sum of feature weights, and averaged across cross-validation folds. For the heatmap in Figure 2, all microbial species that were assigned non-zero weights in at least $80 \%$ of cross-validation folds were included.

\section{Linear mixed effect model}

To test for differential abundance of microbial species between CDI and non-CDI samples while taking into account possible confounding factors, we employed linear mixed effect models as 
implemented in the ImerTest package ${ }^{85}$. After filtering for prevalence (prevalence of at least $5 \%$ in three or more studies), we tested the log-transformed abundance of each microbial species using a linear mixed effect model with "CDI status" as fixed and "Study" and "Age group" as random effects. Effect size and $p$-values were extracted from the model and $p$-values were corrected for multiple hypothesis testing using the Benjamini-Hochberg procedure. In addition, we used a linear mixed effect model to test for a relationship between sequencing depth and the ability to detect $C$. difficile in CDI study populations as well as on the wider global set of samples (considering only dereplicated samples from time series). In this analysis, study was considered as a random effect.

\section{Pathogenic toxin genes detection in metagenomes}

Identification of toxin-codifying genes and their assignment was performed using the VFDB database ${ }^{86}$, as downloaded in March 2021. In our analysis we did not investigate the presence of the binary toxin gene, since this toxin alone has not been associated with disease severity ${ }^{87}$. Mapping against VFDB was performed via BWA $^{88}$ and filtering via NGLess ${ }^{89}$, using $99 \%$ as minimal alignment percentage identity threshold and 75bp as minimal read length match. Toxin gene profiles were computed with gffquant (https:/github.com/grp-bork/gff quantifier, version 1.2.3) using the 1overN count model. Toxin genes included in VFDB refer to the following strains: C. difficile 630, E. coli CFT073, O44:H18 042 and 0157:H7 str. EDL933, S. flexneri 2a str.301, P. aeruginosa PAO1, C. perfringens str.13 and SM101, S. aureus RN4220, subsp. aureus MW2 and N315.

\section{Subspecies analysis}

C. difficile subspecies detection was performed on all $C$. difficile positive samples with MetaSNV2 ${ }^{90}$. Reads from 2441 samples were mapped against the proGenomes $\mathrm{v} 1^{91}$ species representatives genomes for 3 species in the Clostridium genus: i) specl_v2_0051 (NCBI taxonomy ID 272563, PRJNA78) Clostridioides difficile, ii) specl_v2_0052 (NCBI taxonomy ID 1151292, PRJNA85757) Clostridioides difficile and iii) specl_v2_1125 (NCBI taxonomy ID 1408823, PRJNA223331) Clostridioides mangenotii.

By mapping to multiple genomes and only using the uniquely mapped reads, we essentially focus on the species-specific core genomic regions. Mappings that had at least $97 \%$ identity and a match length of at least 45bp were kept. Mapping and filtering was performed using BWA $^{88}$ and NGLess ${ }^{89}$. Reads that mapped uniquely across the 3 reference genomes were used 
to call single nucleotide variants (SNVs) using metaSNV2 with default parameters. Of the initial 2441 samples, 197 passed the requirements for robust SNV calling (8\%) and 179 could be used to detect subspecies presence. Substructure within the population was assessed in the resultant SNV profiles according to previously reported approach ${ }^{88,92}$. Briefly, dissimilarities between samples were calculated based on SNV abundance profiles and the resultant distance matrix was tested for clusters using the Prediction Strength algorithm ${ }^{93}$. Distance matrix is plotted using $\mathrm{R}$ and the pheatmap package with average clustering. Six samples with extreme dissimilarity to all other samples were removed from the distance matrix for illustrative purposes (SAMN08918181, SAMN09980608, SAMN10722477, SAMN13091313, SAMN13091317, SAMN13091322).

\section{Building SNP-tree}

SNPs obtained via metaSNV2 were arranged into an alignment PHY input format file suitable for IQTREE2 tree-builder ${ }^{94}$. Sites which had multiple variants/alleles (in this case only SNPs) were replaced with alternative non-reference allele if they were supported by $>50 \%$ of reads mapped against specl_v2_0051 (NCBI taxonomy ID 272563, PRJNA78), see 'Subspecies analysis' section for mapping details. This results in forced fixing of alleles across polymorphic sites within each sample. To include specl_v2_0052 (NCBI taxonomy ID 1151292, PRJNA85757) Clostridioides difficile and specl_v2_1125 (NCBI taxonomy ID 1408823, PRJNA223331) Clostridioides mangenotii into SNP input file for IQTREE2 we used Mauve ${ }^{95}$ to 1) order contigs of both genomes against specl_v2_0051 genome, 2) align ordered contigs, 3) export SNP profile from Mauve and further merged alignment SNP profile on metaSNV2 output file using matching positions. Missing coverage was kept as gaps in the alignment and tree construction. Following command was used to build phylogenetic tree: iqtree -s <input snp file> -m GTR+ASC -B 1000.

\section{Data availability}

Raw metagenomic sequencing data have been uploaded to the European Nucleotide Archive under the accession number PRJEB50977. The accession numbers relative to the other public studies included in this meta-analysis are available in Supplementary Table 2. The filtered taxonomic profiles and associated metadata used for the analyses are available in 
Supplementary Tables 4-6. The analysis code and commands used in the project are deposited in the GitHub repository at https://github.com/pamela314/cdifficile.

\section{Acknowledgements}

We are thankful to the members of the groups of Peer Bork, Georg Zeller and Ed Kuiper, Prof. Thomas Dandekar (University of Würzburg) and Dr. Athanasios Typas (EMBL) for fruitful discussions. We are also grateful to Lia Oken for her continuous support and her availability for brainstorming sessions. We acknowledge funding from EMBL and the European Research Council (MicrobioS grant no. ERC-AdG-669830 to P.B.). This work comprises results from Pamela Ferretti's doctoral thesis.

\section{Figure \& Table Legends}

Figure 1. (A) On top, fecal metagenomes used in the meta-analysis from 10 public CDI or diarrheal study populations, divided by groups: subjects diagnosed with CDI (CDI, $n=234$ ), subjects diagnosed with other diseases than CDI (D-Ctr, $n=114)$, and healthy subjects ( $\mathrm{H}-\mathrm{Ctr}$, $\mathrm{n}=186$ ) samples. See Methods for further group description. At the bottom, fraction of $C$. difficile positive samples per group. C. difficile toxin genes were found only among CDI $C$. difficile positive samples. (B) Rarefied species richness across the three groups. (C) Prevalence, relative abundance of species that induce antibiotic-associated diarrhea (AAD) among CDI and controls. (D) Prevalence of single and multiple toxigenic species found among $C$. difficile positive and $C$. difficile negative samples, divided by sample group. Mean comparison p-values calculated using Wilcoxon-test.

Figure 2. (A) Microbial species signature associated with CDI, healthy controls and diseased controls, as seen by LASSO model and (B) its associated AUC values for all samples as well as for single study populations, when comparing CDI to healthy and diseased controls combined (left) and CDI with healthy controls only (right). In the latter, the AUC value of Vincent_2016 is left intentionally blank as only diseased controls (D-Ctr) are available for this study population (see Supplementary Table 1).

Figure 3. (A) Overview of the global dataset collection composed of 42,814 samples, from 253 publicly available studies, including host-associated and environmental samples (internal ring), and 
their subcategories (external ring). The human gut was the most represented environment $(66,2 \%$, $n=28,347$ ) in our collection. (B) Stratification of the human gut samples, divided by health status and age group (see Methods for detailed age group description, numbers refer to the number of samples prior to read filtering). (C) Prevalence of $C$. difficile positive samples per category. Total values refer to the number of samples after the initial read filtering and before time series dereplication (see Methods). From this point onwards, results refer to this extended collection of 42,814 samples.

Figure 4. (A) C. difficile prevalence in stool samples in healthy and diseased human (left) and animal (right) hosts over lifetime. C. difficile prevalence divided by (B) health status and delivery mode, (C) health status and prematurity and (D) feeding mode within the first semester of life. For animal samples, only species with at least ten total samples are shown. (E) Species richness of human stool samples, with and without $C$. difficile, across age groups in healthy (left) and diseased subjects (right). See Methods for details on age group definitions. For samples belonging to time series, only the representative samples are included in the estimations (see Methods). Mean comparison p-values calculated using t-test.

Figure 5. Species associated with $C$. difficile, divided by age group and health status: significant positive associations are shown in dark orange, significant negative ones in dark blue ( $p$-values adjusted using $\mathrm{BH}$ ). Lighter shades indicate non significant associations. Only species significantly associated with $C$. difficile in at least one age group are shown. Number of samples per each category shown in the lower part. See Methods for details on age group definitions. Species group separation was not affected by presence of $C$. difficile toxin genes (data not shown). On the right, per species annotation of their i) oxygen requirement (see also Supplementary Figure 12 for the differential enrichment across the two groups) and ii) trend over lifetime. Positive Spearman's rho values indicate species more commonly found in the gut microbiome of healthy adults, negative values the opposite trend.

Supplementary figure 1. (A) Prevalence of $C$. difficile and other antibiotic-associated diarrhea (AAD) species, divided by study. For $C$. difficile only, the portion of samples with toxigenic $C$. difficile is shown (dotted segments). CDI diagnosis procedure is shown on top of each study. Each row represents the diagnostic algorithm (combination of tests) used to diagnose CDI. Tests performed are indicated with black dots (white if not). For example, in "Langdon et al. 2021", CDI was diagnosed if symptoms were present and toxigenic culture for $C$. difficile was positive, or if symptoms were present and the enzymatic immunoassays for $C$. difficile was positive, or if pseudomembranous colitis was identified. Diagnostic protocols abbreviations: "EIA": Enzymatic 
ImmunoAssays, "GDH": glutamate dehydrogenase, "NAAT" nucleic acid amplification test (including PCR), "PMC": pseudomembranous colitis. (B) Prevalence of the number of AAD species (C. difficile not included) identified in each CDI sample, divided by $C$. difficile positivity.

Supplementary Figure 2. Species-level composition for CDI, diseased control and healthy control samples as seen by mOTUs v2.0. Species with minimum relative abundance $\geq 0.01$ and prevalence $\geq 0.1$ are shown.

Supplementary figure 3. Species significantly enriched (in yellow) or depleted (in blue) in terms of relative abundance in CDI compared to diseased and healthy controls, as identified by linear mixed effect model analysis. Species known to cause antibiotic-associated diarrhea (AAD) are highlighted in red. $C$. difficile is not included in the analysis. Study and age group were considered as nuisance variables (and modeled as random effects). To be noted that Enterobacter sp. (ref_mOTU_0036) includes Shigella flexneri and Escherichia coli.

Supplementary figure 4. (A) Adjusted p-values for ANOVA analysis aiming to explain variance in $C$. difficile presence by the available metadata, either as single factors (two top rows) or by a combination of multiple factors (sequential, bottom rows, in the exact order shown along the $X$ axis), in data sets that were broken down by age group (infants versus adults). (B) Geographical distribution across continents and countries for $C$. difficile prevalence in the stools of healthy infants (left) and adults (right). Only countries with $C$. difficile prevalence above $1 \%$ are shown. Prevalence was calculated based on presence/absence of $C$. difficile from mOTUs v2.0 $0^{30}$ taxonomic profiles.

Supplementary figure 5. Prevalence of $C$. difficile across subjects diagnosed with specific diseases (left) and subjects taking antibiotics (right). Average $C$. difficile prevalence is shown with grey bars, age-group specific prevalence is highlighted with colored dots. Number of datasets used for prevalence estimations is reported in the lower part. Abbreviations: NEC: "Necrotizing enterocolitis"; ASCVD: "atherosclerotic cardiovascular disease"; T2D: "Type 2 diabetes"; CRC: "Colorectal cancer"; ADA: "advanced adenoma"; NAA: "non-advanced adenoma". See Methods for details on age group definitions.

Supplementary figure 6. C. difficile prevalence in infants and children, divided by possible combinations of health status, prematurity and delivery mode. 
Supplementary figure 7. Time of the first appearance of $C$. difficile in infants and children timeseries. The grey line indicates the total number of samples per age interval, considering all samples independently of health status, delivery mode, gestational age and diet.

Supplementary figure 8. Community similarity (Bray-Curtis index) of healthy infant-mother pairs in presence or absence of $C$. difficile in stools across the first four years of life. Only full-term infant samples were included.

Supplementary figure 9. Prevalence of $C$. difficile toxin genes in the stools of healthy and diseased subjects over lifetime, based on the detection of either one or both of $C$. difficile Toxin A (TcdA) or Toxin B genes (TcdB) (see Methods for details). Prevalence is calculated on the number of $C$. difficile positive samples. $C$. difficile toxin genes were exclusively found among human stool samples. See Methods for details on age group definitions.

Supplementary figure 10. Alpha diversity calculations in C. difficile positive and negative samples. (A) Species richness in healthy infants with detailed age resolution. (B-C) species richness in infant samples divided by delivery mode, gestational age and health status. (D) Species evenness (as the inverse Simpson index divided by Richness) across age groups and health status. Species richness Continents with at least five $C$. difficile positive samples are shown. The number of samples per group is shown under each boxplot. Mean comparison p-values calculated using t-test. See Methods for details on age group definitions.

Supplementary figure 11. Species with co-occurrence $(\log O R>0)$ or co-exclusion $(\log O R<0)$ pattern with $C$. difficile across gut stool samples from humans $(n=14,095)(A)$ and animals $(n=3,967)(B)$. Labelled species are highlighted in aquamarine and violet, for humans and animals respectively. Clostridium paraputrificum and Clostridium neonatale were among the very few species significantly associated with $C$. difficile in both human and animal stools.

Supplementary Figure 12. Spearman's Rho values for the species listed in Figure 5, across the two groups.

Supplementary Figure 13. SNV similarity across $C$. difficile positive samples from our global metagenomic survey. Tree rooted with Clostridioides mangenotii as outgroup. Missing metadata 
are shown in white. All metagenomes have ANI similarity $>95 \%$. See Methods for detailed analysis description.

Supplementary Table 1. Overview of the public metagenomic CDI study populations included in the CDI meta-analysis, divided by sample group.

Supplementary Table 2. Overview of the public metagenomic studies included in the global meta-analysis.

Supplementary Table 3. Mean C. difficile prevalence and relative abundance estimation across age groups and health status.

Supplementary Table 4-5. Samples metadata before and after timeseries dereplication.

Supplementary Table 6. Samples filtered taxonomic profiles, as provided by mOTUs v2.0. 


\section{References}

1. Hall, I. C. \& O'toole, E. INTESTINAL FLORA IN NEW-BORN INFANTS: WITH A DESCRIPTION OF A NEW PATHOGENIC ANAEROBE, BACILLUS DIFFICILIS. Am. J. Dis. Child. 49, 390-402 (1935).

2. Bartlett, J. G., Moon, N., Chang, T. W., Taylor, N. \& Onderdonk, A. B. Role of Clostridium difficile in antibiotic-associated pseudomembranous colitis. Gastroenterology $75,778-782$ (1978).

3. Voth, D. E. \& Ballard, J. D. Clostridium difficile toxins: mechanism of action and role in disease. Clin. Microbiol. Rev. 18, 247-263 (2005).

4. Zhang, S. et al. Cost of hospital management of Clostridium difficile infection in United States-a meta-analysis and modelling study. BMC Infect. Dis. 16, 447 (2016).

5. CDC. Antibiotic resistance threats in the United States, 2019. (2019) doi:10.15620/cdc:82532.

6. Smits, W. K., Lyras, D., Lacy, D. B., Wilcox, M. H. \& Kuijper, E. J. Clostridium difficile infection. Nat Rev Dis Primers 2, 16020 (2016).

7. Kociolek, L. K. \& Gerding, D. N. Breakthroughs in the treatment and prevention of Clostridium difficile infection. Nat. Rev. Gastroenterol. Hepatol. 13, 150-160 (2016).

8. Tang, D. M., Urrunaga, N. H. \& von Rosenvinge, E. C. Pseudomembranous colitis: Not always Clostridium difficile. Cleve. Clin. J. Med. 83, 361-366 (2016).

9. Chia, J.-H. et al. Clostridium innocuum is a vancomycin-resistant pathogen that may cause antibiotic-associated diarrhoea. Clin. Microbiol. Infect. 24, 1195-1199 (2018).

10. Larcombe, S., Hutton, M. L., Riley, T. V., Abud, H. E. \& Lyras, D. Diverse bacterial species contribute to antibiotic-associated diarrhoea and gastrointestinal damage. J. Infect. 77, 417-426 (2018).

11. Galdys, A. L. et al. Prevalence and duration of asymptomatic Clostridium difficile carriage among healthy subjects in Pittsburgh, Pennsylvania. J. Clin. Microbiol. 52, 2406-2409 
(2014).

12. Kato, H. et al. Colonisation and transmission of Clostridium difficile in healthy individuals examined by PCR ribotyping and pulsed-field gel electrophoresis. J. Med. Microbiol. 50, 720-727 (2001).

13. Rousseau, C. et al. Clostridium difficile carriage in healthy infants in the community: a potential reservoir for pathogenic strains. Clin. Infect. Dis. 55, 1209-1215 (2012).

14. Vincent, C. et al. Bloom and bust: intestinal microbiota dynamics in response to hospital exposures and Clostridium difficile colonization or infection. Microbiome 4, 12 (2016).

15. Smillie, C. S. et al. Strain Tracking Reveals the Determinants of Bacterial Engraftment in the Human Gut Following Fecal Microbiota Transplantation. Cell Host Microbe 23, 229_ 240.e5 (2018).

16. Podlesny, D. \& Florian Fricke, W. Microbial Strain Engraftment, Persistence and Replacement after Fecal Microbiota Transplantation. doi:10.1101/2020.09.29.20203638.

17. Kim, J. et al. Quantitative characterization of Clostridioides difficile population in the gut microbiome of patients with $\mathrm{C}$. difficile infection and their association with clinical factors. Scientific Reports vol. 10 (2020).

18. Langdon, A. et al. Microbiota restoration reduces antibiotic-resistant bacteria gut colonization in patients with recurrent Clostridioides difficile infection from the open-label PUNCH CD study. Genome Med. 13, 28 (2021).

19. Monaghan, T. M. et al. Metagenomics reveals impact of geography and acute diarrheal disease on the Central Indian human gut microbiome. Gut Microbes 12, 1752605 (2020).

20. Stewart, D. B. et al. Integrated Meta-omics Reveals a Fungus-Associated Bacteriome and Distinct Functional Pathways in Clostridioides difficile Infection. mSphere vol. 4 (2019).

21. Kumar, R. et al. Identification of donor microbe species that colonize and persist long term in the recipient after fecal transplant for recurrent Clostridium difficile. npj Biofilms and Microbiomes vol. 3 (2017). 
22. Watson, A. R. et al. Adaptive ecological processes and metabolic independence drive microbial colonization and resilience in the human gut. doi:10.1101/2021.03.02.433653.

23. Milanese, A. et al. Microbial abundance, activity and population genomic profiling with mOTUs2. Nat. Commun. 10, 1014 (2019).

24. Parks, D. H. et al. Evaluation of the Microba Community Profiler for Taxonomic Profiling of Metagenomic Datasets From the Human Gut Microbiome. Front. Microbiol. 0, (2021).

25. Kiu, R. \& Hall, L. J. An update on the human and animal enteric pathogen Clostridium perfringens. Emerg. Microbes Infect. 7, 141 (2018).

26. Zollner-Schwetz, I. et al. Role of Klebsiella oxytoca in antibiotic-associated diarrhea. Clin. Infect. Dis. 47, e74-8 (2008).

27. Högenauer, C., Hammer, H. F., Krejs, G. J. \& Reisinger, E. C. Mechanisms and management of antibiotic-associated diarrhea. Clin. Infect. Dis. 27, 702-710 (1998).

28. Chia, J.-H. et al. Clostridium innocuum is a significant vancomycin-resistant pathogen for extraintestinal clostridial infection. Clin. Microbiol. Infect. 23, 560-566 (2017).

29. Larcombe, S., Hutton, M. L. \& Lyras, D. Involvement of Bacteria Other Than Clostridium difficile in Antibiotic-Associated Diarrhoea. Trends Microbiol. 24, 463-476 (2016).

30. Schmidt, T. S. et al. Extensive transmission of microbes along the gastrointestinal tract. Elife 8, (2019).

31. Golić, N. et al. In vitro and in vivo antagonistic activity of new probiotic culture against Clostridium difficile and Clostridium perfringens. BMC Microbiol. 17, 108 (2017).

32. Na, X. \& Kelly, C. Probiotics in clostridium difficile Infection. J. Clin. Gastroenterol. 45 Suppl, S154-8 (2011).

33. Klare, I. et al. Antimicrobial susceptibilities of Lactobacillus, Pediococcus and Lactococcus human isolates and cultures intended for probiotic or nutritional use. J. Antimicrob. Chemother. 59, 900-912 (2007).

34. Schubert, A. M. et al. Microbiome data distinguish patients with Clostridium difficile infection 
and non-C. difficile-associated diarrhea from healthy controls. MBio 5, e01021-14 (2014).

35. Jangi, S. \& Thomas Lamont, J. Asymptomatic Colonization by Clostridium difficile in Infants: Implications for Disease in Later Life. Journal of Pediatric Gastroenterology \& Nutrition vol. 51 2-7 (2010).

36. Lees, E. A., Miyajima, F., Pirmohamed, M. \& Carrol, E. D. The role of Clostridium difficile in the paediatric and neonatal gut - a narrative review. Eur. J. Clin. Microbiol. Infect. Dis. 35, 1047-1057 (2016).

37. Crobach, M. J. T. et al. Understanding Clostridium difficile Colonization. Clin. Microbiol. Rev. 31, (2018).

38. Deane, J. et al. A multicentre analysis of Clostridium difficile in persons with Cystic Fibrosis demonstrates that carriage may be transient and highly variable with respect to strain and level. J. Infect. 82, 363-370 (2021).

39. Bauer, M. P. et al. Patients with cystic fibrosis have a high carriage rate of non-toxigenic Clostridium difficile. Clin. Microbiol. Infect. 20, O446-9 (2014).

40. McDonald, L. C. et al. Clinical Practice Guidelines for Clostridium difficile Infection in Adults and Children: 2017 Update by the Infectious Diseases Society of America (IDSA) and Society for Healthcare Epidemiology of America (SHEA). Clin. Infect. Dis. 66, e1-e48 (2018).

41. Drall, K. M. et al. Clostridioides difficile Colonization Is Differentially Associated With Gut Microbiome Profiles by Infant Feeding Modality at 3-4 Months of Age. Frontiers in Immunology vol. 10 (2019).

42. Korpela, K. et al. Selective maternal seeding and environment shape the human gut microbiome. Genome Res. 28, 561-568 (2018).

43. Ferretti, P. et al. Mother-to-Infant Microbial Transmission from Different Body Sites Shapes the Developing Infant Gut Microbiome. Cell Host Microbe 24, 133-145.e5 (2018).

44. Thevenon, O., Adema, W. \& Clarke, C. Background brief on fathers' leave and its use. 
http://rgdoi.net/10.13140/RG.2.2.27717.24808 (2016) doi:10.13140/RG.2.2.27717.24808.

45. Daquigan, N., Seekatz, A. M., Greathouse, K. L., Young, V. B. \& White, J. R. Highresolution profiling of the gut microbiome reveals the extent of Clostridium difficile burden. NPJ Biofilms Microbiomes 3, 35 (2017).

46. Seekatz, A. M., Rao, K., Santhosh, K. \& Young, V. B. Dynamics of the fecal microbiome in patients with recurrent and nonrecurrent Clostridium difficile infection. Genome Med. 8, 47 (2016).

47. Zhou, Y. et al. Metagenomic Approach for Identification of the Pathogens Associated with Diarrhea in Stool Specimens. J. Clin. Microbiol. 54, 368-375 (2016).

48. Bartlett, J. G. \& Gerding, D. N. Clinical recognition and diagnosis of Clostridium difficile infection. Clin. Infect. Dis. 46 Suppl 1, S12-8 (2008).

49. Polage, C. R., Solnick, J. V. \& Cohen, S. H. Nosocomial diarrhea: evaluation and treatment of causes other than Clostridium difficile. Clin. Infect. Dis. 55, 982-989 (2012).

50. Jackson, M., Olefson, S., Machan, J. T. \& Kelly, C. R. A high rate of alternative diagnoses in patients referred for presumed Clostridium difficile infection. J. Clin. Gastroenterol. 50, 742-746 (2016).

51. Reich, N. et al. Prospective Review of Clostridioides difficile Testing Indications to Inform Local Laboratory Stewardship Initiatives. Infection Prevention in Practice 1, 100017 (2019).

52. Tang, D. M. et al. Pseudomembranous Colitis: Not Always Caused by Clostridium difficile. Case Rep. Med. 2014, 812704 (2014).

53. Gateau, C., Couturier, J., Coia, J. \& Barbut, F. How to: diagnose infection caused by Clostridium difficile. Clin. Microbiol. Infect. 24, 463-468 (2018).

54. Humphries, R. M., Uslan, D. Z. \& Rubin, Z. Performance of Clostridium difficile toxin enzyme immunoassay and nucleic acid amplification tests stratified by patient disease severity. J. Clin. Microbiol. 51, 869-873 (2013).

55. Burnham, C.-A. D. \& Carroll, K. C. Diagnosis of Clostridium difficile infection: an ongoing 
conundrum for clinicians and for clinical laboratories. Clin. Microbiol. Rev. 26, 604-630 (2013).

56. Lee, H. S., Plechot, K., Gohil, S. \& Le, J. Clostridium difficile: Diagnosis and the Consequence of Over Diagnosis. Infect Dis Ther 10, 687-697 (2021).

57. Crobach, M. J. T. et al. European Society of Clinical Microbiology and Infectious Diseases: update of the diagnostic guidance document for Clostridium difficile infection. Clin. Microbiol. Infect. 22 Suppl 4, S63-81 (2016).

58. Tenover, F. C., Baron, E. J., Peterson, L. R. \& Persing, D. H. Laboratory diagnosis of Clostridium difficile infection can molecular amplification methods move us out of uncertainty? J. Mol. Diagn. 13, 573-582 (2011).

59. Rodriguez, C. et al. Laboratory identification of anaerobic bacteria isolated on Clostridium difficile selective medium. Acta Microbiol. Immunol. Hung. 63, 171-184 (2016).

60. Martínez-Meléndez, A. et al. Current knowledge on the laboratory diagnosis of Clostridium difficile infection. World J. Gastroenterol. 23, 1552-1567 (2017).

61. Litvin, M. et al. Identification of a pseudo-outbreak of Clostridium difficile infection (CDI) and the effect of repeated testing, sensitivity, and specificity on perceived prevalence of CDI. Infect. Control Hosp. Epidemiol. 30, 1166-1171 (2009).

62. Website. 'BBLTM Clostridium Difficile Selective Agar.' n.d. Accessed October 21, 2021. https://www.bd.com/resource.aspx?IDX=20564.

63. Parks, T., Chapman, T., Culver, E. \& Scarborough, M. Persistent diarrhoea after presumed Clostridium difficile infection at Oxford Radcliffe Hospitals NHS Trust: an unrecognised entity. Gut 60, A72-A72 (2011).

64. Polage, C. R. et al. Overdiagnosis of Clostridium difficile Infection in the Molecular Test Era. JAMA Intern. Med. 175, 1792-1801 (2015).

65. Hensgens, M. P. M. et al. Diarrhoea in general practice: when should a Clostridium difficile infection be considered? Results of a nested case-control study. Clin. Microbiol. Infect. 20, 
O1067-74 (2014).

66. Spina, A. et al. Spectrum of enteropathogens detected by the FilmArray GI Panel in a multicentre study of community-acquired gastroenteritis. Clin. Microbiol. Infect. 21, 719-728 (2015).

67. Bäckhed, F. et al. Dynamics and Stabilization of the Human Gut Microbiome during the First Year of Life. Cell Host Microbe 17, 852 (2015).

68. Yassour, M. et al. Natural history of the infant gut microbiome and impact of antibiotic treatment on bacterial strain diversity and stability. Sci. Transl. Med. 8, 343ra81 (2016).

69. Chu, D. M. et al. Maturation of the infant microbiome community structure and function across multiple body sites and in relation to mode of delivery. Nat. Med. 23, 314-326 (2017).

70. Rodríguez, J. M. et al. The composition of the gut microbiota throughout life, with an emphasis on early life. Microb. Ecol. Health Dis. 26, 26050 (2015).

71. Rigottier-Gois, L. Dysbiosis in inflammatory bowel diseases: the oxygen hypothesis. ISME J. 7, 1256-1261 (2013).

72. Rivera-Chávez, F. et al. Depletion of Butyrate-Producing Clostridia from the Gut Microbiota Drives an Aerobic Luminal Expansion of Salmonella. Cell Host Microbe 19, 443-454 (2016).

73. Kubota, H. et al. Longitudinal Investigation of Carriage Rates, Counts, and Genotypes of Toxigenic Clostridium difficile in Early Infancy. Appl. Environ. Microbiol. 82, 5806-5814 (2016).

74. Schutze, G. E., Willoughby, R. E., Committee on Infectious Diseases \& American Academy of Pediatrics. Clostridium difficile infection in infants and children. Pediatrics 131, 196-200 (2013).

75. Davis, M. Y., Zhang, H., Brannan, L. E., Carman, R. J. \& Boone, J. H. Rapid change of fecal microbiome and disappearance of Clostridium difficile in a colonized infant after 
transition from breast milk to cow milk. Microbiome 4, 53 (2016).

76. Chang, T. W., Sullivan, N. M. \& Wilkins, T. D. Insusceptibility of fetal intestinal mucosa and fetal cells to Clostridium difficile toxins. Zhongguo Yao Li Xue Bao 7, 448-453 (1986).

77. Keel, M. K. \& Songer, J. G. The distribution and density of Clostridium difficile toxin receptors on the intestinal mucosa of neonatal pigs. Vet. Pathol. 44, 814-822 (2007).

78. Eglow, R. et al. Diminished Clostridium difficile toxin A sensitivity in newborn rabbit ileum is associated with decreased toxin A receptor. J. Clin. Invest. 90, 822-829 (1992).

79. Cornick, S., Tawiah, A. \& Chadee, K. Roles and regulation of the mucus barrier in the gut. Tissue Barriers 3, e982426 (2015).

80. Willemsen, L. E. M., Koetsier, M. A., van Deventer, S. J. H. \& van Tol, E. A. F. Short chain fatty acids stimulate epithelial mucin 2 expression through differential effects on prostaglandin $\mathrm{E}(1)$ and $\mathrm{E}(2)$ production by intestinal myofibroblasts. Gut 52, 1442-1447 (2003).

81. Pruitt, R. N. \& Lacy, D. B. Toward a structural understanding of Clostridium difficile toxins A and B. Front. Cell. Infect. Microbiol. 2, 28 (2012).

82. Felczykowska, A., Krajewska, A., Zielińska, S. \& Łoś, J. M. Sampling, metadata and DNA extraction - important steps in metagenomic studies. Acta Biochim. Pol. 62, 151-160 (2015).

83. Coelho, L. P. et al. Towards the biogeography of prokaryotic genes. Nature (2021) doi:10.1038/s41586-021-04233-4.

84. Wirbel, J. et al. Microbiome meta-analysis and cross-disease comparison enabled by the SIAMCAT machine learning toolbox. Genome Biol. 22, 93 (2021).

85. Kuznetsova, A., Brockhoff, P. B. \& Christensen, R. H. B. ImerTest Package: Tests in Linear Mixed Effects Models. Journal of Statistical Software vol. 82 (2017).

86. Liu, B., Zheng, D., Jin, Q., Chen, L. \& Yang, J. VFDB 2019: a comparative pathogenomic platform with an interactive web interface. Nucleic Acids Res. 47, D687-D692 (2019). 
87. Goldenberg, S. D. \& French, G. L. Lack of association of tcdC type and binary toxin status with disease severity and outcome in toxigenic Clostridium difficile. J. Infect. 62, 355-362 (2011).

88. Li, H. \& Durbin, R. Fast and accurate short read alignment with Burrows-Wheeler transform. Bioinformatics 25, 1754-1760 (2009).

89. Coelho, L. P. et al. NG-meta-profiler: fast processing of metagenomes using NGLess, a domain-specific language. Microbiome 7, 84 (2019).

90. Van Rossum, T. et al. metaSNV v2: detection of SNVs and subspecies in prokaryotic metagenomes. Bioinformatics (2021) doi:10.1093/bioinformatics/btab789.

91. Mende, D. R. et al. proGenomes: a resource for consistent functional and taxonomic annotations of prokaryotic genomes. Nucleic Acids Res. 45, D529-D534 (2017).

92. Costea, P. I. et al. Subspecies in the global human gut microbiome. Mol. Syst. Biol. 13, 960 (2017).

93. Yuan, Q., Guo, X., Ren, Y., Wen, X. \& Gao, L. Cluster correlation based method for IncRNA-disease association prediction. BMC Bioinformatics 21, 180 (2020).

94. Minh, B. Q. et al. IQ-TREE 2: New Models and Efficient Methods for Phylogenetic Inference in the Genomic Era. Mol. Biol. Evol. 37, 1530-1534 (2020).

95. Darling, A. C. E., Mau, B., Blattner, F. R. \& Perna, N. T. Mauve: multiple alignment of conserved genomic sequence with rearrangements. Genome Res. 14, 1394-1403 (2004). 
A 534 WGs Stool Samples 10 Study populations Subjects diagnosed

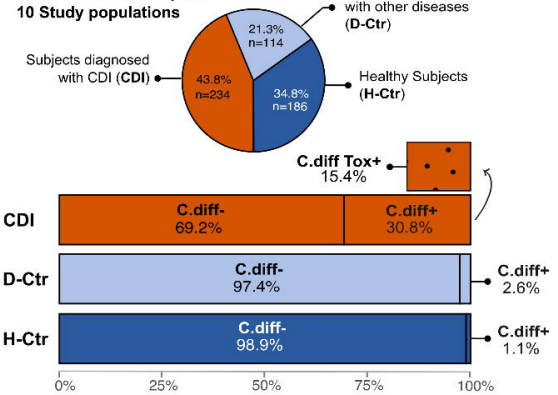

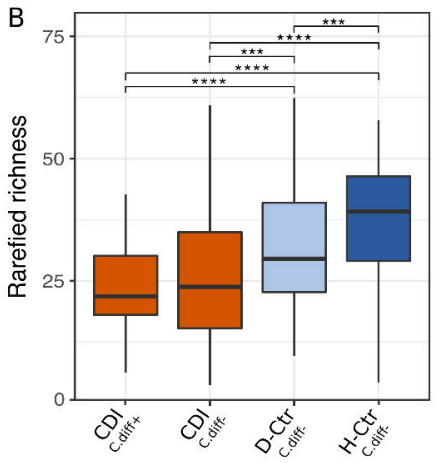

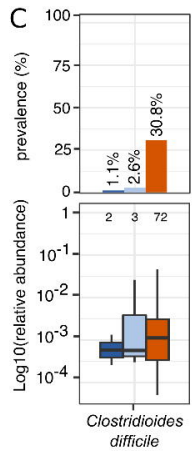

D

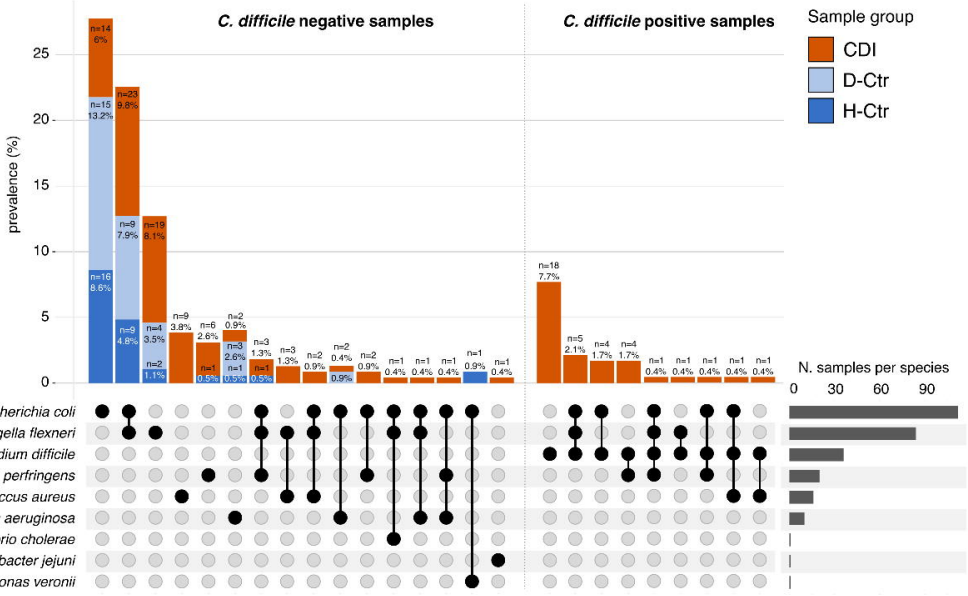


A

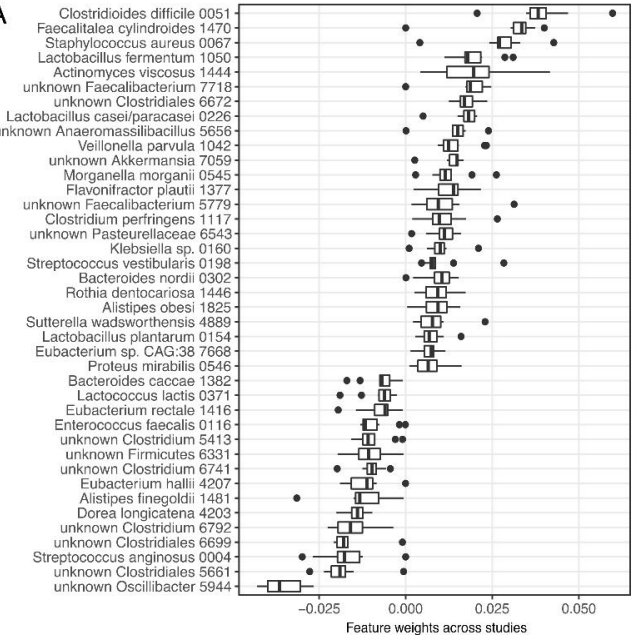

B L LASSO model including $c$. difficile

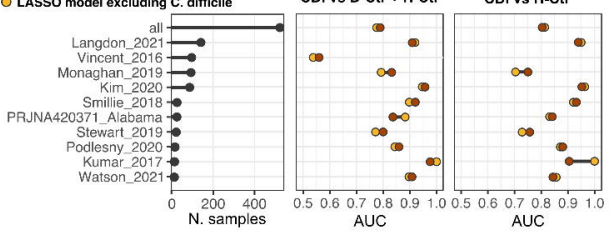

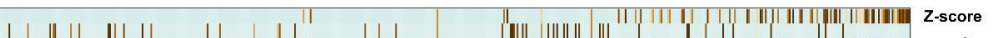

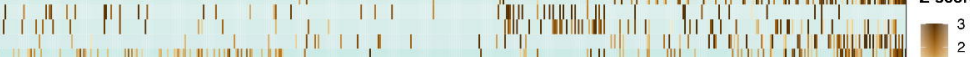

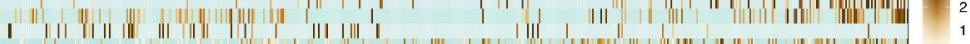

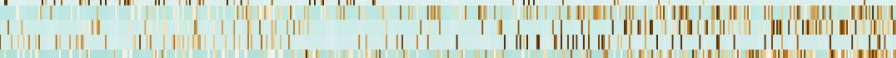
1. +

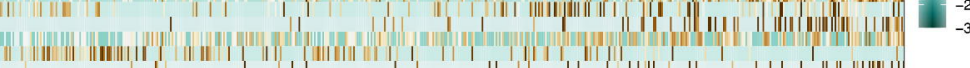

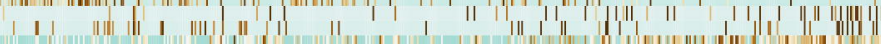

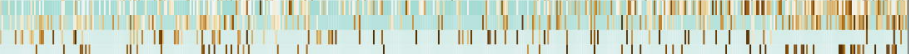

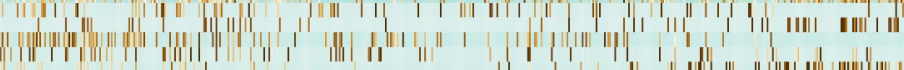

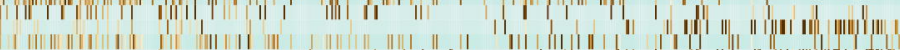

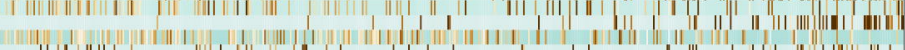

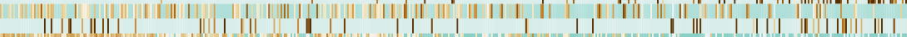
级

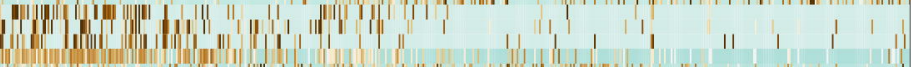

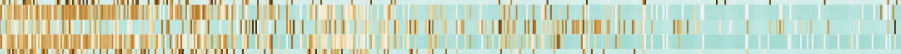

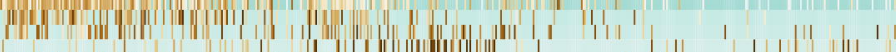

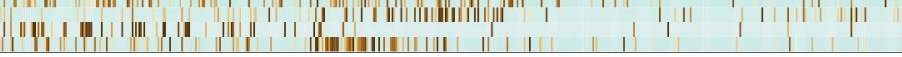

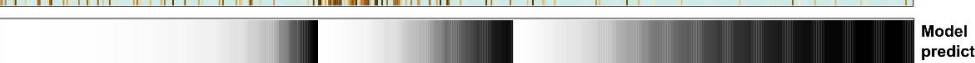

Model

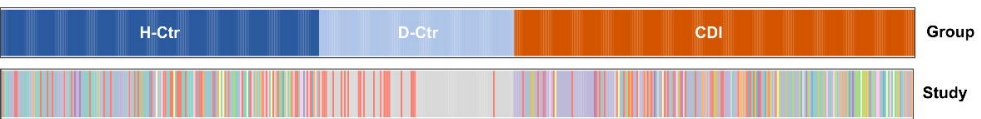

Study

Kim_2020

Kurnar_2017

Langdon_2021

Monaghan_2019

Podlesny_2020

PRJNA420371_Alabama

Smillie_2018

Stewart_2019

Vincent_2016

Watson_2021 

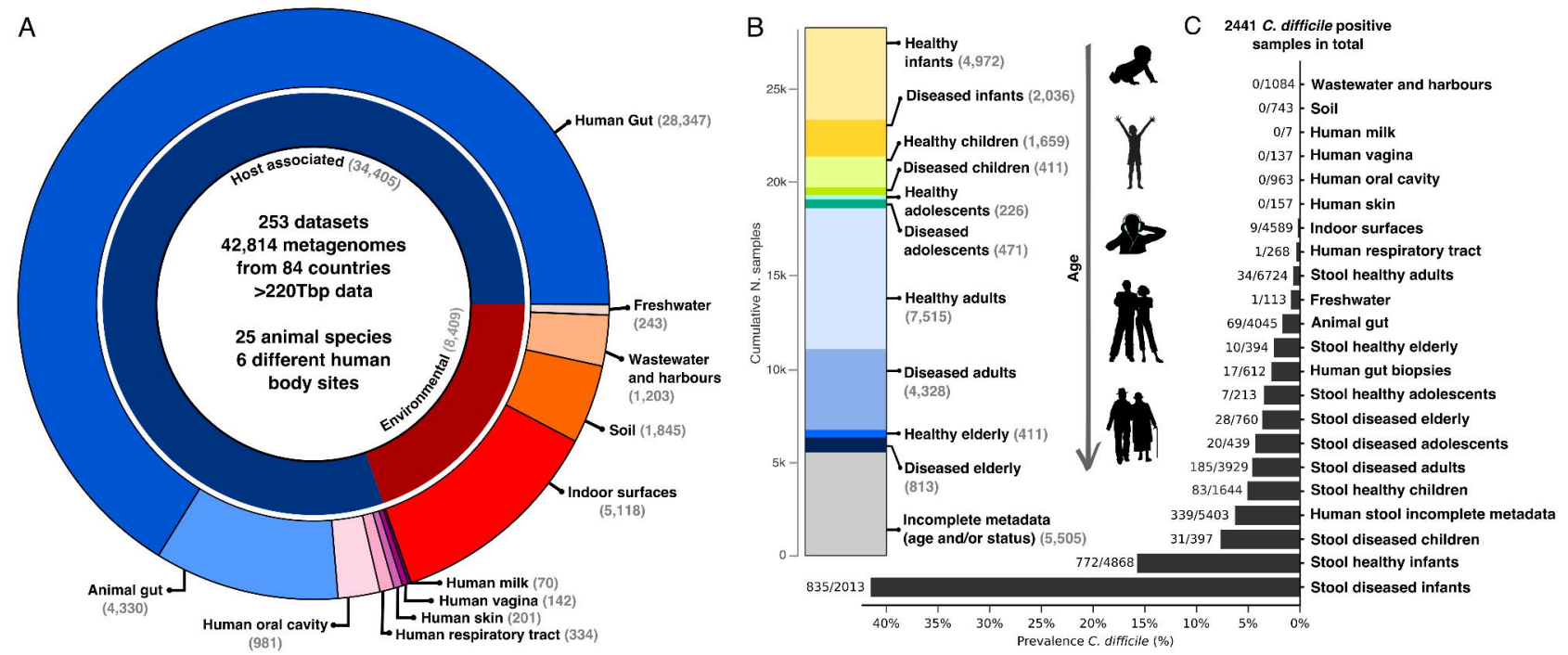


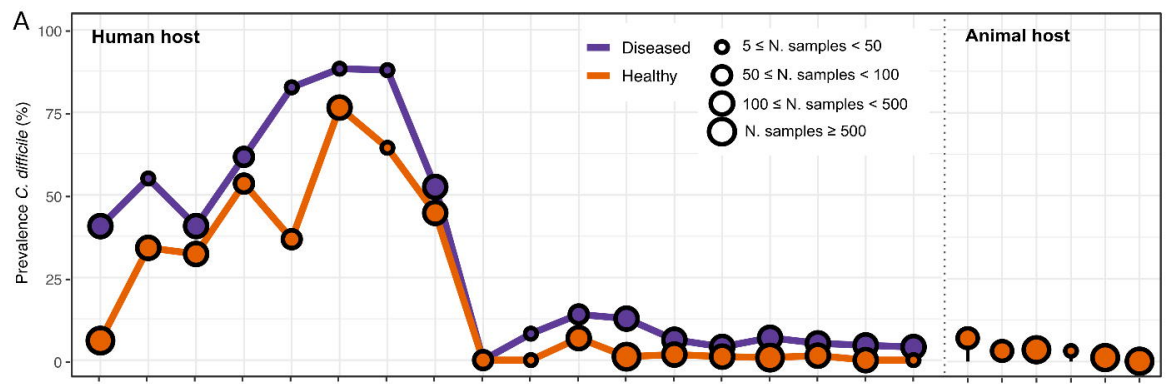

B

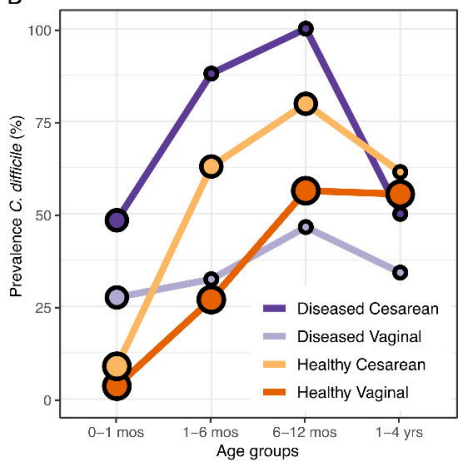

Age groups

C

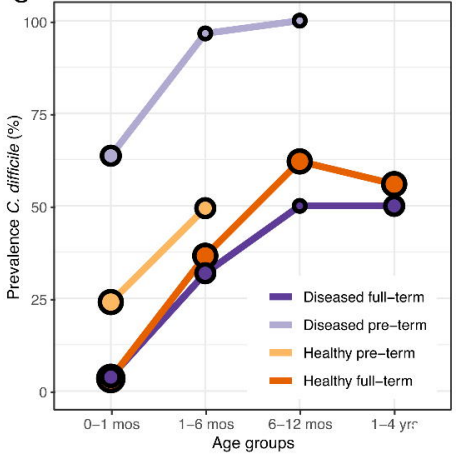

Host species

D

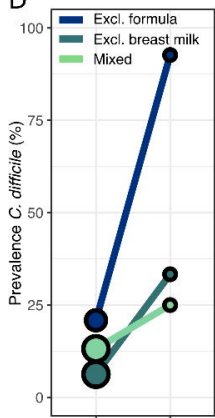

0-1 mos 2-6 mos Age groups

E

Healthy

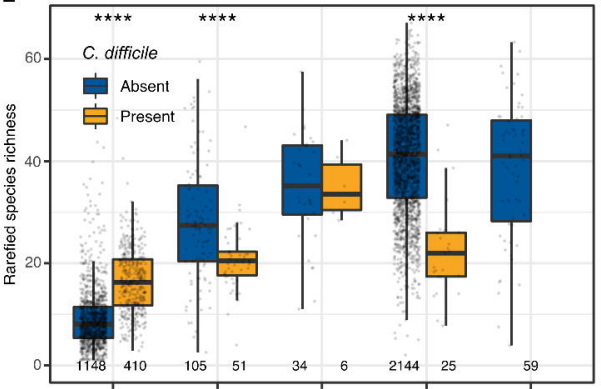

Infant Child Adolescent Adult

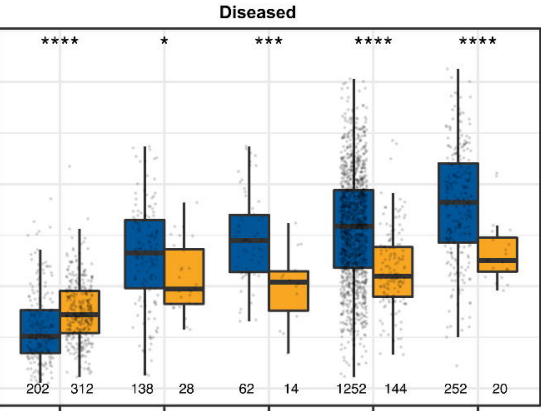


Citrobacter sp. [ref_0076] Klebsiella michiganensis/oxytoca [ref 0079] Bificobacterium breve [ref_0157] Lactobacillus mamnosus [ref 0048] Clostridium perfringens [ref_1117] Enterococcus faecium [ref_0372] Klebsiella sp. [ref_0160]

Streptococcus pneumoniae [ref_0020] Streptococcus pseudopneumoniae [ref 0021] Streptococcus mitis [ref_0028] Enterococcus faecalis [ref 0116] Stroptococcus mitis [ret 0032] Veillonella parvula [ref_1042] unknown Veillonella [meta 5811] Veillonella atypica [ref_0561]

Veillonella dispar [ret 4469 ]

Anaerococcus obesiensis/vaginalis [ref_0429]

Clostridium paraputriticum [ref_2890]

Niameybacter massiliensis [meta 7610] Clostridiales bacterium VE202-09 [ref_2688]

Clostridium innocuum [ref 0643

Blautia producta [ref_1889]

Enterococcus sp. [ref 0674]

Megasphaere micronuciformis [ref 4B40]

Ruminococcus gnavus [ref_0280]

Coprococcus sp. [ref_0303]

Anaerostipes caccae [ref_1381]

Tyzzerella nexilis [ret 4366]

Citrobacter amalonaticus [ref_0042]

Finegoldia magna [ref_1208]

Finegoldia megna [ret 4423]

Enterobacter sp. [ref_0265]

Enterococcus avium [ref_0599]

Erysipelotrichaceae sp. [ref_0885]

Clostridium butyricum [ref_0978]

Clostridium neonatale [meta 5695 ]

Clostridium sp. 7_2_43FAA [ref_4288]

Enterocaccus sp. [ref_1043]

Robinsoniella peoriensis [ref 1234]

Clostridium cadaveris [ref_2891] Clostridiales bacterium VE202-21 [ref 2690] Subdoligranulum sp. 4_3_54A2FAA [ref_4738]

Turicibacter sanguinis [ref_1493]

unknown Bacilli [meta 5934]

unknown Clostridium [meta_6741]

unknown Clostridiales [meta 6885]

unknown Peptostreptococcaceae [meta_7331] Lachnosp raceae bacterium 3_1_57FAA CT 1 [ref_0887]

Parasutterella excrementihominis [ref_1405] Dialister invisus [ref_4598]

Clostridium citroniae [ref 4882]

unknown Clostridiales [meta_6561] Eubacterium sp. CAG:38 [meta_7668]

Roseburia intestinalis [ref_1427]

Ruminococcus torques [ref 1376 ]

Akkermansia muciniphila [ref 1301]

Roseburia hominis [ref_4572]

unknown Clostridiales [meta_6602]

Anaerostipes hadrus [ref_1309]

Blauta sp. KLE 1732 [ref 0859]

Ruminococcus torques [ref_4718]

Blautia wexlerae [ref_0466]

unknown Lachnospiraceae [meta_6937]

Roseburia inulinivorans [ref_4632]

unknown Clostridiales [meta_5712]

butyrate-producing bacterium SS3/4 [ref_3825]

Eubacterium eligens [ref 4389]

Faecalibacterium prausnitzil [ref_4875]

Ruminococcus bromii [ref 4720]

unknown Roseburia [meta_5354]

Eubacterium sulci [ref 5112]

Clostridium sp. [ref_0378]

Clostridium sporosphaeroides [ref_1909]

Varibaculum cambriense [ref_2210]

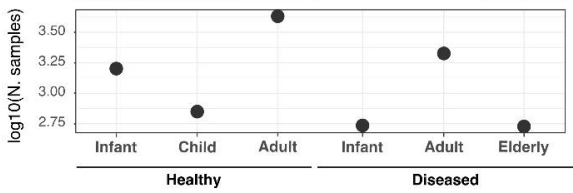

\section{0 \\ 08 \\ ᄀ Association with $C$. difficile}

00

- 0

00

00

00

00

00

○ 0

00

00

00

00

00

00

00

00

00

00

00

00

00

00

00

00

00

00

00

00

00

00

00

00

00

00

00

00

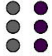

00

00

00

00

ᄀ

Positive $\mathrm{p}<.05$

Positive $p \geq .05$

Neutral

$-$

$7 \square$

Negative $p \geq .05$

]

]

$-$

万

$-7$

을 -

o

\}

$\exists$

$\exists$

$\exists$

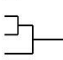

Negative $p<.05$

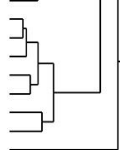

-

-

了

万
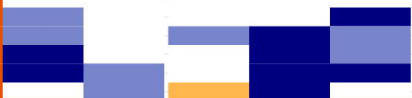

89

08

0

$\circ 8$

ב

$\sim 7$

윽

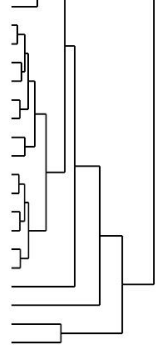

0

ii) Trend over lifetime (Spearman's rho)

$\begin{array}{lll}-1.0 & 0 & 1.0\end{array}$

\section{i) Oxygen requirement class}

Facultative anaerobic or aerobic

Obligate anaerobic

Unknown 